\title{
Robust Short Message Protocol
}

\author{
Vitaly U. Klimashov, RSFLabs
}

\begin{abstract}
Nowadays, the software and hardware solutions based on Linux OS and open-source software became popular not only for server or desktop use; they are also widely employed by the developers of the distributed control and telemetry systems, including the mission critical ones. The goal of our research is design and implementation of a reliable point-to-point message transmission protocol.
\end{abstract}

ISO - International Organization for Standardization, RSMP - Robust Short Message Protocol,

RTT - Round-Trip delay Time,

TCP - Transmission Control Protocol, UDP - User Datagram Protocol

\section{I.INTRODUCTION}

$\mathrm{M}$ odern computer communication systems are crucial for different kinds of military, government and civil applications. Evergrowing demand for automated control and management systems results in growing requirements; especially strict are the robustness requirements for the communication systems that operate in harsh conditions and under heavy load. The same requirements apply for many other different kinds of applications and systems that rely heavily on the data transfer layer. In a modern dynamic world where the computer networks appear and disappear fast, the task of their interconnection is especially important, as well as the common standardized interprocess communication protocols.

Some large commercial organizations develop their own protocols to suit their specific needs. Those protocols are often designed to be used with certain kinds of hardware and software, so that their range of possible applications is narrowed severely.

\section{II.Problem Analysis}

Our company had taken part in the design and implementation of a geographically distributed remote control system where the messages were transmitted over Internet. Some of the key requirements were: robustness,

Manuscript received March 26, 2009.

A. A. Emelyanov is an engineer of RSFLabs, Russia, N.Novgorod, 603104, Nartova, 6. phone: +7831-278-90-47 url: www.rsflabs.biz

R. M. Dmitrienko is an engineer of RSFLabs, Russia, N.Novgorod, 603104, Nartova, 6. phone: +7831-278-90-47 url: www.rsflabs.biz

V. U. Klimashov is an engineer of RSFLabs, Russia, N.Novgorod, 603104, Nartova, 6. phone: +7831-278-90-47 url: www.rsflabs.biz

V. V. Mokhin is an engineer of RSFLabs, Russia, N.Novgorod, 603104, Nartova, 6. phone: +7831-278-90-47 url: www.rsflabs.biz noise immunity, guaranteed data delivery and highest performance possible. At first it was decided to employ TCP for telemetry and remote control messages transmission because of its wide recognition and known robustness. But during the design phase of the project it turned out that most of the target nodes are located in the distant areas far from large settlements and hence out of the zone of reliable Internet access. The actual quality of the available data transmission channels was extremely poor: lost packet rate up to $30 \%$, average transmission delay up to $300 \mathrm{~ms}$. It became obvious that TCP didn't quite suit our needs. While providing robust, guaranteed data transfer, it actually had very poor performance because of large amount of auxiliary data being sent. As the delivery time was of our greatest priority, we were forced to cease using TCP. Such harsh working conditions forced us to investigate profoundly available data transmission protocols in order to find one that would suit our specific needs.

The most widespread protocols, such as TCP and UDP, are standardized by ISO. The developers are allowed to employ them freely whenever needed. At the same time, most of those protocols have quite strict limitations and thus are not suitable for use with low quality data transmission channels. Protocols governed by ISO are fundamental and generic by nature, and do not perform well under extreme conditions. In such cases, adaptation of the protocols is required.

\section{III.Proposed Solution And Current Status}

In order to solve the problem of reliable data transmission over low-quality data transmission channels we had decided to develop a new protocol based on UDP, which is fast enough to suit our needs of building the real time distributed systems. We had to extend UDP with certain features in order to meet the requirements, i.e. guaranteed data delivery and automatic protocol self-adaptation upon channel quality changes.

The protocol is of peer-to-peer kind. All of the packets being sent are grouped into sessions. The packet structure is based on the UDP packet structure; the only difference is the additional 1-octet field that carries the sequence number of the packet. Such numbering allows for lost packet detection and retransmission on demand. Instead of sending every packet once, it was decided to use preventive retransmission: every packet is sent in a given number of copies called "a pack.” Sequence numbering and preventive retransmission are the basic functions used to achieve guaranteed data delivery. Packet structure is depicted at the figure 1. 


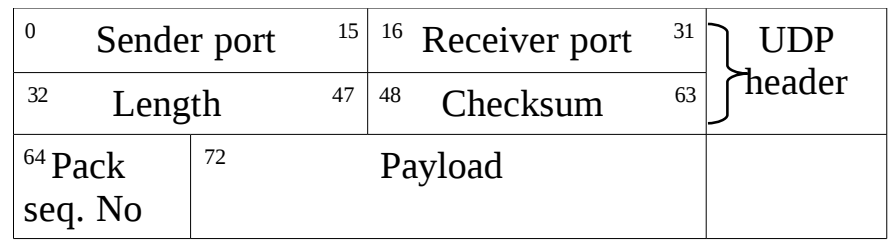

Figure 1. RSMP packet

Auxiliary messages are also implemented. They perform connection establishment and release functions, as well as a few others (retransmission request, lost packs detection, etc). Unlike data messages, their payload is predefined: it consists of command codes and auxiliary data whenever needed. The structure of auxiliary messages is depicted at the figure 2 .

\begin{tabular}{|c|c|c|c|c|c|c|c|}
\hline 0 & \multicolumn{2}{|c|}{ Sender port } & 15 & 16 & Receiver port & 31 & \multirow{2}{*}{$\begin{array}{c}\text { UDP } \\
\text { header }\end{array}$} \\
\hline 32 & \multicolumn{2}{|c|}{ Length } & 47 & 48 & Checksum & 63 & \\
\hline & $x 00^{71}$ & ${ }^{72} \mathrm{Msg}$ & & 81 & Auxiliary data & & \\
\hline
\end{tabular}

Figure 2. Auxiliary RSMP packet

Data transfer itself is based on the UDP protocol. Hence, we've achieved relatively high operating speed if compared to TCP. At the same time, our extensions provide for guaranteed data delivery.

This protocol was named RSMP (Robust Short Message Protocol). It is intended to provide reliable data transfer service with the lowest delays possible over the low-quality data transfer channels with significant packet loss and RTT. It is supposed that the protocol should be employed for short telemetry and remote control messages transmission in the distributed monitoring and control systems.

Its primary advantages are:

- guaranteed data delivery;

- $\quad$ high performance;

- automatic self-adaptation upon channel quality changes

We have performed extensive testing of the implemented protocol. It was confirmed that the software developed is able to perform well in harsh conditions. The software was tested and is already used in the several parts of the geographically distributed telemetry and remote control systems located in Volgograd and Nizhny Novgorod. The protocol was proven to provide reliable service when used with low-quality data transmission channels with random delays and packet loss.

\section{IV.Future Prospect}

The RSMP protocol is supposed to be employed widely as a part of distributed remote monitoring and control systems. Its features allow for building reliable telemetry and telecontrol systems even in the distant rural regions. 